\title{
Unterstützung für Menschen in Not - eine ureigene medizinische Aufgabe
}

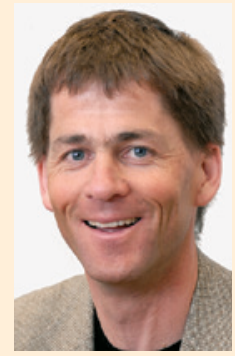

Liebe Kolleginnen und Kollegen,

wie macht man es denn am besten richtig? Dieser Gedanke ging mir durch den Kopf, als ich bei einer Vorstandssitzung einer großen deutschen Patientenselbsthilfeorganisation den Vortrag des Fundraising-Teams sah und die letzte Folie die „Gefahr“ des Erdbebens in Nepal betonte. Denn damit könnte die Spendenbereitschaft für deutsche Patienten zurückgehen...

Die Not ist jedoch weltweit groß und gerade in Nepal machen die Menschen eine sehr schwere Zeit durch. Die mediale Berichterstattung war nur kurz vorhanden und damit war auch die Spendenbereitschaft vermutlich nur kurz etwas höher. In Nepal ist aber mit den beiden Erdbeben gerade in wichtigen Touristengegenden (Pokhare, Kathmandu, SoluKhumbu, Langtang, Rolwalling) sehr viel zerstört worden und der Wiederaufbau wird nicht nur Monate, sondern Jahre dauern. Daher ist es unbedingt notwendig, dass hier dauerhafte Hilfe für dieses Land mit kaum vorhandenen eigenen Ressourcen geleistet wird. Auch wenn am Beginn der Berichterstattung sicher einiges an Spendengeldern geflossen ist, müssen weiter Spenden dabei helfen, den Menschen vor Ort die Existenz zu sichern. Es sind ja nicht nur Wohnhäuser und Stallungen sowie Scheunen zerstört worden, sondern auch Lodges, Hotels und Wege wie Brücken. Damit ist die zentrale Einkommensquelle des Landes empfindlich getroffen und viele Reisestornierungen zeigen in diese Richtung. Aus meiner Sicht sollten wir aber nicht stornieren, sondern trotzdem nach Nepal fahren und versuchen, den Menschen vor Ort mit Kaufkraft und vielleicht auch tatkräftiger Hilfe unter die Arme zu greifen. Dies ist sicher ganz im Sinne der Nepali (siehe z.B. die Homepage des Kathmandu Guest House www.ktmgh.com).

Da viele nicht wissen, wem sie am besten spenden sollen, empfehle ich beispielsweise die Seite der BExMed, www.bexmed.de, hier sind unter Aktuelles die Links von Organisationen aufgeführt, die verlässlich und ohne Verwaltungsverluste vor Ort Hilfe leisten.

Diese Hilfe haben aber nicht nur die Menschen in Nepal und in anderen Entwicklungsländern nötig, sondern gerade auch die Menschen, die mit ihrer Not nach oft traumatischer Fluchterfahrung nach Deutschland kommen. In 3 Beiträgen in diesem Heft ist dargestellt, welche Probleme hierbei auftauchen und welche Lösungsmöglichkeiten bestehen. Dabei wird auch deutlich, dass zum Beispiel mit HIV und Tuberkulose ein Wandel in den typischen Krankheitsbildern einhergeht, der die behandelnden Ärzte vor besondere Herausforderungen in Diagnostik und Therapie stellt.

Herausforderungen also in so vielen Bereichen, und wie sollen wir es jetzt am besten richtig machen? Ich empfehle, dem eigenen Gefühl zu folgen und so viel zu geben, wie es einem selbst möglich ist, denn nur wer gibt, dem wird gegeben.

Mit herzlichen Grüßen aus München Prof. Dr. Rainald Fischer 